\title{
Toxocariasis: A Practical Approach to Management of Ocular Disease
}

\author{
W. J. DINNING*, S. H GILLESPIE †, R. J. COOLING*, R. M. MAIZELS $\ddagger$ \\ London
}

\section{Summary:}

The diagnosis of ocular toxocariasis is difficult to make with certainty. New methods of antigen detection are helpful. Surgical intervention may play a role in improving the prognosis, but the place of specific chemotherapy remains undefined. We discuss three case histories to illustrate these points. We suggest that the visual prognosis need not be so poor as commonly believed, and outline a suggested plan of management.

Toxocara infection is common in the United Kingdom. $2.6 \%$ of adults have specific antitoxocaral antibodies ${ }^{1}$ and surveys in children show higher incidence. ${ }^{2}$ The figure of $2.6 \%$ may seem low, but it has been calculated that at least 16,000 new cases of infection are occurring each year in the United Kingdom. ${ }^{3}$ High prevalence of seropositivity is found in adults at special risk, for example $15 \%$ of dog breeders in the U.K. ${ }^{4}$ and $25 \%$ of hydatid disease control officers in New Zealand. ${ }^{5}$ Many Toxocara infections are completely asymptomatic ${ }^{6}$ but ocular involvement, when it occurs, may have devastating consequences. About 300 clinical cases are reported each year in the United Kingdom, of whom $50 \%$ have evidence of ocular involvement. $^{7}$

The place of chemotherapy in the treatment of ocular disease is not well established, with few reports in the literature. The prognosis is generally considered to be poor. ${ }^{8} \mathrm{We}$ report the clinical features and the course of treatment in three children with ocular toxocariasis.

\section{Case Histories}

Case 1. A 14 year old healthy boy presented with a 6 week history of blurring in the left eye. Acuity was hand movements. There was a subretinal inflammatory lesion in the upper temporal quadrant, with vitreoretinal traction producing a band running to the optic disc (Fig. 1). There was a dense cellular infiltration of the vitreous.

Anti-toxocara antibodies were found in serum and at higher levels in ocular fluid. Toxocara antigen was demonstrated in the vitreous and aqueous by a 2 -site capture ELISA using a Toxocara canis specific monoclonal antibody 9 .

$\mathrm{He}$ was treated with thiabendazole $(50 \mathrm{mg} / \mathrm{Kg} /$ day) and oral prednisolone for 7 days. Vitrectomy and epiretinal dissection was performed to clear the vitreous and relieve retinal traction.

Acuity improved over 3 months to $6 / 36$, and more gradually to $6 / 24$. The eye remains free of signs of inflammation, and the retinal picture is stable.

Case 2. A 4 year old girl was found to have acuity of hand movements in the right eye on routine testing in a screening clinic. There were signs of chronic anterior and posterior segment inflammmation. In the temporal retinal periphery there was a small flat choroidoretinal scar, from 
which a tunnel-shaped vitreous condensation ran to the disc.

Anti-toxocara antibodies were found at high levels in the serum. There was moderate eosinophilia.

While under treatment with topical steroids and mydriatics the eye became much more inflamed. Thiabendazole was given, together with subtenons Depomedrone injection.

The inflammation settled completely, the ocular media cleared, and the acuity has improved to $6 /$ 24.

Case 3. A 6 year old boy complained of sudden pain and a shadow in his right eye. Acuity was 6/ 60. The aqueous was clear, but there were cells in the vitreous. The initial clinical picture was of

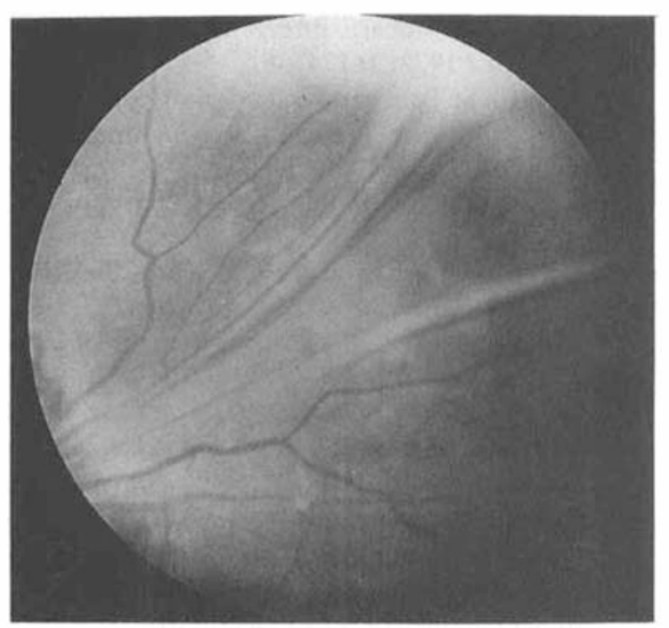

Fig 1. Vitreoretinal traction in case 1

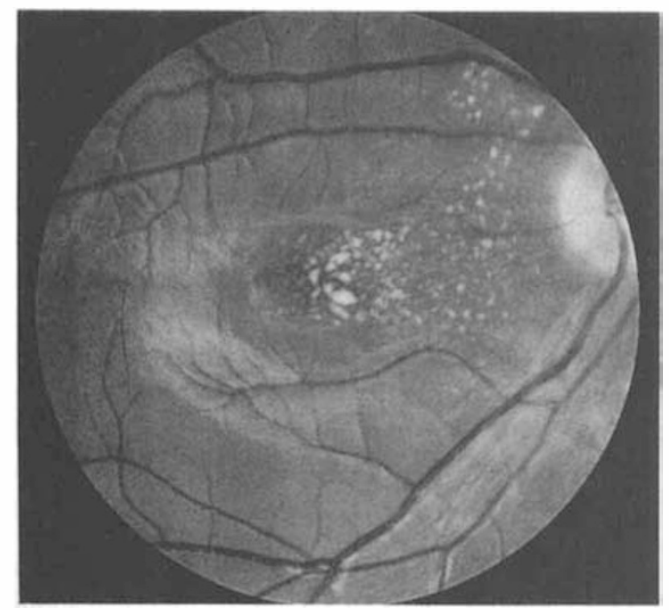

Fig 2. Posterior pole of case 3 optic disc oedema, and he was treated immediately with oral predisolone. Within a few days the macular oedema and perimacular exudates could be seen (Fig. 2).

High levels of anti-toxocara antibodies were found in the serum; toxocara antigen was also found in the serum. There was also an eosinophilia.

Thiabendazole was added to the treatment for 7 days. The acuity improved to 6/12. All signs of inflammation settled, but structural changes remain at the macula.

\section{Discussion}

Human toxocariasis has two characteristic syndromes, visceral larva migrans (VLM), and ocular disease. Signs and symptoms of VLM are fever, hepatomegaly, splenomegaly, urticarial or nodular skin rash, abdominal pain, bronchospasm and convulsions.

Three clinical pictures of ocular disease are generally described:

(1) a raised lesion at or near the posterior pole

(2) a peripheral granuloma involving retina, with overlying vitreous opacification and vitreoretinal traction (fig.1)

(3) diffuse endophthalmitis

Other findings, such as papillitis, are reported. The frequency with which tests for $T$. canis are requested in patients with "uveitis" and found to be positive suggests that the spectrum of ocular inflammation caused by this organism may be wider than traditionally believed.

Definitive diagnosis may be made by the histological examination of biopsy specimens. However, it is rarely clinically justifiable to obtain this material. The parasite may be difficult to find on biopsy. ${ }^{10}$ The diagnosis of VLM can easily be made using a combination of eosinophil count, total immunoglobulin level and the presence of iso-haemagglutinins $^{11}$. Specific diagnosis can be confirmed by ELISA for antibodies directed against the excretory/secretory (ES) antigens ${ }^{1}$. The diagnosis of ocular disease is more difficult. Many of these patients have raised levels of antitoxocaral antibody, but they are usually lower than in VLM ${ }^{12}$. Antitoxocaral antibodies may be detected in aqueous and vitreous. The presence of eosinophils in the 
aqueous provides strong support for the diagnosis.

The detection of toxocara antigen in aqueous, vitreous or serum samples was of value in two of these cases. This represents a useful advance in confirming the diagnosis.

These cases demonstrate that the visual prognosis need not be so serious as is commonly imagined. In particular, the use of recently developed surgical techniques permits the salvage of eyes that would previously have suffered irreversible retinal damage from scarring. All three cases were improved following treatment, but the individual contribution of steroids and specific chemotherapy cannot be determined. However, this outcome is better than might be predicted from previous series ${ }^{8}$. There is a reluctance to treat cases of Toxocara infection because the drugs utilised are relatively toxic and unproven in controlled trial. In addition, the diagnosis could never be firm using antibody detection methods alone. With the benefit of antigen detection to confirm the diagnosis and monitor the results of therapy, further studies are in progress, to explore the possibility of a wider clinical spectrum for the ocular disease and to determine the ideal treatment regime.

\section{Conclusion}

We currently propose the following plan of management:

(1) Eye disease alone:

local and periocular or systemic steroids surgery where appropriate

(2) Eye disease alone, which does not respond to (1):

add specific antihelminthic and continue systemic steroids:

thiabendazole $50 \mathrm{mg} / \mathrm{Kg} /$ day for 7 days

$$
+
$$

prednisolone $0.5-1 \mathrm{mg} / \mathrm{Kg} /$ day

(3) Eye disease with VLM, or high antibody

levels:

local steroids and mydriatics

$$
+
$$

thiabendazole and systemic steroids as in (2) from the outset.
The authors wish to thank Mr. R. Dalgleish for permission to examine and report case 2, and Dr. A. Voller and Mr. B. Robertson for help with serological diagnosis.

\section{References}

${ }^{1}$ De Savigny DH, Voller A, Woodruff AW: Toxocariasis: serological diagnosis by enzyme immunoassay. J Clin Pathol 1979; 32: 284-8.

2 Josephs DS, Bhinder P, and Thompson AR: The prevalence of toxocara infection in a child population. Public Health (London) 1981; 95: 273-5.

${ }^{3}$ Baxter DN and Leck I: The deleterious effects of dogs on human health: 2. Canine zoonoses. Community Med. 1984; 6: 185-97.

${ }^{4}$ Woodruff AW, De Savigny DH, Jacobs DE: Study of Toxocaral infection in dog breeders. Br Med J 1978; 2: 1747-8.

5 Clemett RS: Toxocaral infection in hydatid control officers: diagnosis by enzyme immunoassay. N.Z Med J 1985; 98: 737-9.

${ }^{6}$ Bass JL, Mehta KA, Glickman LT, Eppes BM: Clinically inapparent Toxocara infection in children. N Eng J Med 1983; 308: 723-4.

7 Rée GH, Voller A, Rowland HAK: Toxocariasis in the British Isles. $\mathrm{Br}$ Med $J$ 1981; 228: 628-9.

${ }^{8}$ Molk R: Ocular toxocariasis: A review of the literature. Ann Ophthalmol 1983; 15: 216-31.

${ }^{9}$ Robertson BD, Burkot TR, Gillespie SH, Kennedy MW, Wambai Z, Maizels RM: Detection of circulating parasite antigen and specific antibody in Toxocara canis infections. (submitted for publication).

${ }^{10}$ Woodruff AW: Toxocariasis. Br Med J 1970: 3: 663-9.

${ }^{11}$ Huntley CC, Costas MC, Lyerly A: Visceral larva migrans syndrome: clinical characteristics and immunological studies. Paediatr. 1965; 35: 523-6.

12 Biglan AW, Glickman LT, Lobes LA: Serum and vitreous Toxocara antibody in nematode endophthalmitis. Am J Ophthalmol 1979; 88: 898-901. 